\title{
LÄRM, MONTAGE UND RHYTHMUS. URBANE PRINZIPIEN POPULÄRER MUSIK
}

\author{
Malte Friedrich
}

\begin{abstract}
»We believed that music is nothing but organized noise.
You can take anything - street sounds, us talking, whatever you want - and make it music by organizing it."

(Shocklee, Produzent von Public Enemy)
\end{abstract}

Lange Zeit galten Städte ausschließlich als materielle Artefakte, die zwar von ihren Bewohnern sehr unterschiedlich wahrgenommen wurden, sich aber letztlich aus der räumlichen Aufteilung und der gebauten Umwelt ableiten ließen. Aber Städte sind nicht nur gebaute Umwelten, sondern sie werden auch durch Repräsentationen konstituiert. Darunter fallen genauso die srealistischen Darstellungen und Beschreibungen von Städten wie künstlerische Auseinandersetzungen oder utopische Entwürfe. Stadt ist nicht nur ein materieller Ort, sondern immer auch eine imaginäre Umwelt, in der es, wie Victor Burgin feststellt, zu einer Vermischung des Materiellen, Erfundenen und Erlebten kommt:

»The city in our actual experience is at the same time an actually existing physical environment, and a city in a novel, a film, a photograph, a city seen on television, a city in a comic strip, a city in a pie chart and so on « (Burgin 1996: 28; Hervorhebung im Original).

Der Einfluss von Repräsentationen für die Erschaffung von Städten nimmt in postindustriellen Gesellschaften aus drei Gründen noch weiter zu. Erstens kommt es zu einer televisionären Beobachtung von Stadträumen durch Stadtverwaltung, Stadtforschung und private Institutionen, die zu einer kontinuierlichen Doppelung von Ausschnitten der Stadt führt. Sie dient einer kontinuierlichen Steuerung und Kontrolle möglichst aller Abläufe in der Stadt und der Normierung und Disziplinierung der Bevölkerung, indem die Beobachtungsinstrumente dazu eingesetzt werden, >problematische < Stadt- 
gebiete und ihre Bewohner zu überwachen und generell abweichendes Verhalten zu verhindern oder zumindest einzudämmen (Blum 2003).

Ein zweiter Grund für den Bedeutungszuwachs von Stadtrepräsentationen sind die technischen Medien. In Zeitschriften, Büchern, Fernsehen, Filmen oder im Internet sind die Stadt und die in ihr anzutreffenden Praktiken äußerst prominent vertreten. Genauso ist die Stadt nicht nur in fast jedem HipHop-Video zu sehen, sondern auch generell in den aktuellen urbanen Soul-Videos. Was heute als urban gilt, ist nicht länger nur alleine an städtische Orte gekoppelt, selbst wenn diese auch weiterhin für die Bildung neuer Szenen wichtig sind. Urbane Kultur findet genauso in den Medien statt, und durch die Adaption der durch sie mitproduzierten oder -geprägten urbanen Praktiken ist sie umgekehrt oft an den unterschiedlichsten Orten auf der ganzen Welt anzutreffen.

Ein dritter Grund für den Anstieg der Bedeutung von Repräsentationen ist ironischerweise die prägende Kraft medialer Stadtdarstellungen für die Gestaltung von Städten. Es ist schon lange nicht mehr nur Las Vegas, welches in eine reine Simulationswelt transformiert wird, oft unter Zuhilfenahme von Themen aus Hollywood-Filmen, sondern ein genereller Trend, der dazu führt, zumindest einzelne Stadtgebiete in Europa und den USA zu eventisieren, in ästhetisch ansprechende und möglichst perfekt aussehende Orte zu transformieren, die im Endergebnis aussehen wie Computeranimationen oder Bilder aus Hochglanzmagazinen. So werden die Städte für den touristischen Blick gestaltet und die alten Industrie- und Speicherorte zu touristischen Attraktionen umfunktioniert.

\section{Visuelle Repräsentationen der Stadt}

Auffallend an den Thesen zur Repräsentation ist, dass sie sich ausschließlich auf visuelle Medien beziehen. Die für die moderne Stadt schon von Georg Simmel (1995: 727f.) aufgestellte These der Dominanz des Sehsinns über die anderen Sinne wird so nicht nur bestätigt, sondern sogar radikalisiert. Die Stadt ist danach nur noch als eine Text- oder Bilderwelt zu bestimmen. In diesem Zusammenhang hat James Donald (1999: 92) vorgeschlagen, die Stadt nicht als Ort, sondern als eine spezielle Art des Sehens zu charakterisieren, in der sich urbane Praktiken mit medialen Repräsentationen vermischen.

Bestimmt man aber die Stadt auf diese Weise, dann sind die weiteren, nicht-visuellen Sinneseindrücke nicht nur dem Sehen untergeordnet, sondern müssen sogar als irrelevant eingeschätzt werden. Diese Implikation des 
Arguments steht im krassen Gegensatz zur Bedeutung des Hörens in der Stadt, die sich alleine am hohen Stellenwert des Musikhörens an privaten und öffentlichen Orten zeigt. Die Stadt mag zwar ein Ort des Schweigens sein, darauf hatten Simmels Überlegungen zur Dominanz des Sehens hinweisen wollen, aber sie ist auch ein Ort des kontinuierlichen Musikkonsums. Die These von der Dominanz des Sehsinns greift daher zu kurz und kann keine Erklärung zur Wirkungsweise der auditiven Welt der Stadt anbieten.

\section{Das Problem der fehlenden Denotation von Musik}

Nicht nur Musikhören steht in enger Verbindung mit der Stadt, sondern viele Musikstile entstehen in ihr (Gillett 1996; George 2002). Besonders in der populären Musik entfalten sich in Städten immer wieder neue Stile, die sich an Stadtbewohner richten, deren Lebensweise reflektieren und gleichzeitig mit hervorbringen. So wäre es naheliegend, die Frage zu stellen, ob nicht auch deren Musik Städte repräsentieren kann und zu deren Konstitution beiträgt. Auffälligerweise bezieht sich aber die Literatur bei der Betrachtung von Musik als Repräsentationsmedium und als Medium der Imagination fast ausschließlich auf die Kleidungsstile, die Praktiken und - wenn vorhanden auf die Songtexte. Über die Musik selbst ist zumeist wenig bis nichts zu erfahren. ${ }^{1}$

Dafür gibt es zwei Gründe: Erstens ist die Analyse von Musik aus sozialund kulturwissenschaftlicher Sicht aufwändig und, noch wichtiger, wenig anschlussfähig an die Theorien zur Erklärung kultureller Phänomene. Mit anderen Worten: nicht so sehr die Stadt erweist sich als ein rein visuell geprägtes Gebilde, sondern ein Großteil der Theorien zur Beschreibung des Sozialen fokussiert alleine auf visuelle Phänomene und klammert die weiteren Sinnesdimensionen aus. Zweitens gilt Musik, im Gegensatz zu Texten und Bildern, als nicht denotativ. Anstatt auf etwas außerhalb von sich hinzudeuten, könne man in der Musik vielmehr eine selbstbezügliche Verweisstruktur beobachten. Helmut Rösing hält aus diesem Grund Filme jeder musikalischen Repräsentation überlegen:

»Ein Film wie >Night on Earth von Jim Jarmusch dagegen kann auf der visuellen Ebene urbane Räume weit präziser wiedergeben als jede noch so illustrative Soundcollage, von ihrer Transformation und Verdichtung im Medium einmal ganz zu schweigen « (Rösing 1999: 120).

1 Als Beispiel unter vielen kann die berühmte Studie von Dick Hebdige (1979) zur Subkultur dienen, in der zwar viel über Kleidungsstile, wenig aber über die Musik der von ihm untersuchten Musikszenen zu erfahren ist. 
Noch radikaler argumentiert Peter Martin (1995), der die Bedeutungsgenerierung ausschließlich in den jeweiligen Kontexten, in denen die Musik gehört wird, verortet. Problematisch ist diese alleinige Zuschreibung, weil sie Musik nur noch als einen völlig leeren Container bestimmen kann. Selbst wenn man aber zugesteht, dass Musik eine deutlich höhere Bedeutungsvariabilität gegenüber Texten oder Bildern besitzt, bleibt es unplausibel, der Musik jeglichen Einfluss auf die Generierung von bestimmten Bedeutungen abzusprechen. Es kommt hinzu, dass Musik, genauso wie andere kulturelle Artefakte, in bestimmten sozialen Kontexten entsteht, in denen auch Codes bereitgestellt werden, über die überhaupt erst die Bedeutung des kulturellen Artefakts erzeugt werden kann. Diese Codes vorausgesetzt, kommt es zur Entstehung von bestimmten Bedeutungen einzelner Musikstücke, die sich auf die zu hörenden Klänge oder ihre Verweisstruktur beziehen und insofern bedeutungsgenerierend sind.

\section{Peter Kivys These der strukturellen Isomorphie zwischen Musik und Umwelt}

Peter Kivy geht noch einen Schritt weiter. Für ihn kann Musik durchaus etwas repräsentieren. Dafür nennt Kivy zwei Bedingungen. Erstens muss der Komponist - Kivy denkt ausschließlich an die europäische Kunstmusik - die Intention verfolgen, etwas mit seiner Musik darzustellen oder zu illustrieren, und zweitens muss es zumindest eine strukturelle Isomorphie zwischen der Musik und dem geben, was dargestellt oder illustriert wird:

»What is needed, then, for representation in any kind of detail, of a structure or system of elements, is another structure or system of elements that can more or less be isomorphic with it. For this reason it is musical structure that most often plays the leading part in musical presentation, for it is usually an object or phenomenon with a complex structure of its own that is the subject of the musical representation. For this reason, too, it can now be seen why the simple perceptual properties of sound must play a secondary representational role. Of themselves they do not possess the structure - the differentiation of parts necessary for the representation of complex objects or phenomena « (Kivy 1980: 73, Hervorhebungen im Original).

Erst das komplexe Spiel einzelner Elemente der Musik macht es laut Kivy möglich, von einer Repräsentation zu sprechen. Ein wichtiger Bereich von Repräsentation sind für Kivy Gefühle. Kivy entwickelt den zentralen Gedanken, dass Musik nicht Ausdruck von Gefühlen, sondern stattdessen gefühlsausdrückend (expressive of emotions) sei (Kivy 1980: 14, 2001: 31-49). Musik 
könne so Dinge repräsentieren, indem sie die Gefühle ausdrücke, die in ihrer Gegenwart entstehen (oder entstehen können):

»We need not, that is to say, understand the musical representation of a mountain as involving the impossible task of making me feel the way I would feel if I saw the mountain: music can only do that by sheerest accident. What music can do, perhaps, is be expressive of the emotions one might feel in contemplating a mountain, or (more important) be expressive of the same emotions that the mountain might be expressive of (since mountains, I presume, like weeping willows, or the faces of Saint Bernards, can, and often do posses expressive properties). The theory of musical representation as musical expression, then, in its optimal form, is the theory of musical representation as musical expressiveness: that music represents things by being expressive either of the emotions that those things might arouse in us if we were experiencing them face to face, or by being expressive of the emotions those things are expressive of, or both« (Kivy 1991: 133).

Dieses plausible Argument kann hervorragend auch auf die Illustration von Städtischem durch Musik bezogen werden. Kivys zwei Bedingungen stellen aber für die populäre Musik fast nicht zu überwindende Hürden da. Bis auf wenige Ausnahmen lassen sich nicht ohne Weiteres populäre Musikstücke finden, in denen sich eindeutig oder zweifelsfrei nachweisen lässt, dass die Musiker oder Produzenten mit ihrem Stück eine Repräsentation im Sinne von Kivy mit ihrer Musik erreichen wollen. Und auch die tonale und strukturelle Komplexität, die er für eine Grundbedingung der strukturellen Isomorphie ansieht, ist in populärer Musik zumeist weniger relevant als in europäischer Kunstmusik. Die Sprachähnlichkeit von Musik, die Kivy stillschweigend unterstellt, ist in der populären Musik nicht ohne Weiteres gegeben. So ist es nahezu unmöglich, nach der Repräsentation von Stadt in der populären Musik zu suchen, wenn nur auf die musikalische Struktur eines Stücks alleine geschaut wird. ${ }^{2}$

2 Ein interessanter Versuch, doch einen Zusammenhang zwischen der tonalen Struktur populärer Musikstücke und der Stadt zu analysieren, findet sich bei Krims (2007). 


\section{Korrespondenzen zur Stadt auf der Ebene ästhetischer Prinzipien}

Es gibt aber keinen Grund, warum spezifische Eigenschaften populärer Musik nicht auch repräsentative Funktionen im Sinne von Kivy übernehmen können. Hilfreich ist hier ein einfacher wie plausibler Vorschlag zum Wirkungszusammenhang von bildender Kunst und städtischer Umgebung. Danach manifestiert sich die Wirkung des städtischen Umfelds am wenigsten in der einfachen Darstellung ihrer Veränderungen in Bildern. Die neue IndustrieStadt, so Graham Gilloch (1996: 133), bedurfte auch einer neuen Art der Repräsentation und neuen Sensibilität und Praxis der Künstler, die nur so eine Korrespondenz mit der urbanen Umwelt herstellen konnten (vgl. auch Neumeyer 1999: 241). Wichtiger als der Inhalt der Darstellung ist die Art und Weise, in der dies geschieht - mit anderen Worten die Form, welche die Darstellung annimmt. Arnold Hauser weist deshalb schon Mitte der 1960er Jahre zu Recht darauf hin, dass die neuen Erfahrungen der Stadträume Anfang des 20. Jahrhunderts auch neue Formen der Malerei nach sich zogen, »weil der Impressionismus die Welt mit den Augen des Städters sieht und auf die Eindrücke von außen mit den überspannten Nerven des modernen technischen Menschen reagiert. Er ist ein Großstadtstil, weil er die Wandelbarkeit, den nervösen Rhythmus, die plötzlichen, scharfen, sich aber zugleich wieder verwischenden Eindrücke des städtischen Lebens schildert« (Hauser 1967: 929).

Diese Überlegung lässt sich auch auf die populäre Musik übertragen: Die Beeinflussung und damit der Verweis auf die Stadt lässt sich am besten auf der formalen Ebene finden. Ein Gedanke, der den Überlegungen Theodor W. Adornos (2003: 16) nicht unähnlich ist, da auch dieser Repräsentationen auf der formalen Ebene verortet. Aber entgegen Adorno wird mit Hauser von einer direkten empirischen Beeinflussung der ästhetischen Prinzipien von Musik durch die städtische Umwelt ausgegangen, und nicht eine obskure, weil ohne Medium auskommende, Verbindung von materiell bestimmtem Weltgeist und Musik unterstellt (DeNora 2003: 13f.; Ludwig 2004: 29). Plausibel ist eine solche Verbindung zwischen städtischem Umfeld und Musik, weil nicht nur die Musiker vieler populärer Musikstile oft in Städten leben, sondern auch das Publikum, an das sich die Musik in erster Instanz richtet. 


\section{Lärm}

Obwohl die auditiven Umwelten einzelner Städte äußerst komplex sind, sich aus ganz unterschiedlichen Klängen zusammensetzen und örtliche und zeitliche Differenzierungen aufweisen, bleibt eine Eigenschaft von Städten ihr zentrales Merkmal: Lärm (Schafer 1994: 71-99). Dafür gibt es drei Gründe. Erstens sind Stadtbewohner oft unfreiwillig Klängen bzw. Geräuschen ausgesetzt, die deshalb als lärmend empfunden werden. Zweitens finden sich in der Stadt viele Klänge, allen voran die von Autos, die sich nicht eindeutigen Tonhöhen zuordnen lassen. Und drittens ist die Stadt lärmend, weil sich eine Vielzahl von Klängen übereinander schiebt und so einen relativ konturlosen und kakophonen Klangteppich bildet. Nicht zuletzt tragen die diversen Musikanlagen, über die Musik in der Stadt gehört wird, dazu bei, die Stadt lärmender und lauter zu machen.

Die Klage über zu laute Städte ist nicht neu, im Gegenteil, sie prägt die Stadt schon seit langem (ebd.: 189-191). Die industrialisierten und medialisierten Städte haben aber ein lärmendes Klangumfeld zu einem kontinuierlich präsenten Phänomen gemacht.

Schon Anfang des 20. Jahrhunderts reagierte die Musik der Futuristen auf diese Entwicklung, indem sie versuchte, den Lärm selber in die Musik zu integrieren. Sie war damit stilprägend für die gesamte Entwicklung der Kunstmusik, in der die Grenzen zwischen Ton und Geräusch und Musik und anderen Klängen kritisch hinterfragt oder unterwandert wurden (Fellerer 1989: 219-269). Was diesen Versuchen zumeist fehlte, war aber die Koppelung an die Vorstellungswelten der in den städtischen Umgebungen Lebenden.

Diese Anbindung stellt aber die populäre Musik her. Nicht zufällig ist Lärm auch ein Kennzeichen bestimmter Spielrichtungen der populären Musik. Auffallend oft stammen diese Musikstile aus Städten: Ob Jazz, Rock'n'Roll, Beat, Punk, HipHop oder Techno, sie alle gelten oder galten zumindest zum Zeitpunkt ihrer Entstehung als besonders lärmend.

Nicht nur im Punk, sondern auch im etwa zeitgleich entstehenden HipHop und dem sich Mitte bis Ende der 1980er Jahre entwickelnden Techno werden Geräusche als musikalisches Material genutzt. So hat sich in der DJMusik des HipHop mit dem Scratchen, dem schnellen Hin-und-Her-Bewegen einer Schallplatte, die das ursprüngliche Klangmaterial verändert und variiert, eine eigenständige Technik etabliert, um Geräusche zu erzeugen. Und auch im Techno gehören Geräusche, die z.B. an aufeinander schlagende Metalle von Maschinen oder Generatoren erinnern, zum Standardrepertoire. 
Was zudem in den Stilen Punk, HipHop und Techno die Geräuschhaftigkeit verstärkt, ist das Zurückdrängen der Bedeutung von Harmonie und Melodie. Dies resultiert zum einen aus der hohen Relevanz des Rhythmus, zum anderen im Falle von Punk aus der geringen Einhaltung von Tonhöhen beim Singen, und im Falle von HipHop und Techno aus der Nutzung von Melodiefragmenten, die durch ihre kontinuierliche Wiederholung und Synchronisierung zu weiteren Elementen des Rhythmus werden und nicht länger als eigenständige Melodie erscheinen. So sind Geräusche, neben dem Rhythmus, das konstitutive musikalische Element in Punk, HipHop und Techno. Und als ästhetisches Prinzip korrespondieren sie mit dem lärmenden Stadtraum, in dem sie entstehen.

\section{Montage}

Ein zweites Element, das starke Verbindungen zur Stadt aufweist, ist das im HipHop und Techno angewandte Montageprinzip. Die Montage, das Zusammenfügen unterschiedlicher, nicht unbedingt in einem direkten Zusammenhang stehender Elemente in einem neuen Musikstück, stellt keine neue Erfindung dar. Zum einen werden schon seit langem unterschiedliche Musikstücke im Potpourri hintereinander gesetzt, zum anderen erlaubt die seit Mitte der 1960er Jahre immer aufwändigere Studiotechnik die Fragmentierung des Aufnahmeprozesses, sodass die einzelnen Elemente eines Musikstücks nicht länger zeitgleich aufgenommen werden müssen und das fertige Musikstück eine Montage der einzelnen Aufnahmeschritte darstellt (Behrens 1996: 133; Gracyk 1996). Aber in den meisten Fällen ist es der Endzweck dieser Produktion, den Eindruck zu erwecken, die Musik wäre zu einem einzigen Zeitpunkt eingespielt worden. Die Montage soll also gerade nicht als solche gehört werden (Middleton 1990: 65f.).

Eine andere Form der Montage, in der die Schichtung einzelner musikalischer Ebenen nicht alleine dazu dient, ein Zusammenspiel zu erzeugen, sondern in der die einzelnen Dimensionen gleichzeitig als disparate Elemente erhalten bleiben, ist im Techno und HipHop zu beobachten (Krims 2003). Die Montage dient erstens - nicht unähnlich dem Potpourri - bei Club-Abenden dazu, einzelne vorproduzierte Tracks so miteinander zu vermischen, dass neue Musik entsteht, wie Sabine Vogt feststellt:

»[Die DJs] spielen die Songs nicht nacheinander ab, sondern zerlegen die Strophenstruktur des Songs auf der Schallplatte in einzelne Tracks und justieren diese Tracks in die Horizontale des gleichförmigen Vierviertelschlags, des so genannten Four-to-Floor-Beats. Die Tracks gehen unmerklich ineinander 
über, sodass sich eine schier endlose Klangkulisse ergibt. [Es kommt zum] Aneinanderschichten von vorproduzierten Tracks zu neuen Kombinationen, die das impulsartige Wechselspiel von Klangfarben, Rhythmen und Lautstärken möglich machen« (Vogt 2005: 90f.).

Genauso werden im HipHop einzelne Tracks vom DJ so geschickt ineinander gemischt, dass aus alten Tracks neue Musikstücke entstehen. Das Besondere ist dabei, dass teilweise die ursprünglichen Fragmente, aus denen dieses neue Klangerlebnis erzeugt wird, noch für den Hörer als eigene musikalische Einheiten zu erkennen sind. Dabei dienen aber - hier hört die Ähnlichkeit zum Potpourri auf - die alten Elemente nur als Baustoffe für ein neues Musikstück.

Darüber hinaus ist die Montagetechnik das zentrale Prinzip jedes einzelnen Techno- oder HipHop-Tracks. Dabei werden die einzelnen Schichten des Tracks übereinander gelegt. Sie folgen nicht der in der populären Musik häufig anzutreffenden Grundstruktur aus Melodie plus Begleitung und einer Trennung zwischen Strophe und Refrain. Stattdessen sind die Stücke aus einzelnen Loops und ihren Variationen zusammengebaut, die entweder aus einem perkussiven Rhythmuspattern, einem Ton oder einer Geräuschfolge bestehen. Als Ausgangsmaterial dienen Schallplatten oder selbst eingespielte Klänge, die im Sampler bearbeitet und kombiniert werden. Im HipHop kommen oft noch Raps hinzu, in denen wiederum häufig über das Leben in den Innenstädten berichtet wird. Cheryl L. Keyes beschreibt das Ergebnis dieser Montagetechnik durch das Sampling:

»Through digital sampling, DJs fuse various timbres and textures: voluminous bass sounds (bass guitar and drum), strident piercing sounds (e.g. high glissandi sounds), static >noise<, harmonic dissonance, and a battery of vocal ornamentations from James Brown's yells, grunts, moans, and shouts to speech excerpts« (Keyes 2002: 145).

Für das Sampling und die Montagetechniken im Techno und HipHop sind zwei Deutungen angeboten worden. Richard Shusterman hat vorgeschlagen, Rap als postmoderne Ästhetik zu beschreiben:

»[R]ecycling appropriation rather than unique originative creation, the eclectic mixing of styles, the enthusiastic embracing of the new technology and mass culture, the challenging of modernist notions of aesthetic autonomy and artistic purity, and an emphasis on the localized and temporal rather then the putatively universal and eternal« (Shusterman 1992: 202).

Mit der Enteignung bestehender Musik und ihrer Zusammenstellung in einer neuen Verbindung, in der die disparatesten Musikstile kombiniert würden, sei der Begriff der Autorschaft in Frage gestellt, das Prinzip der entfrem- 
denden Zitation über das der Originalität gestellt. In Anlehnung an Baudrillard nennt Shusterman (ebd.: 205) dies eine Aneignung von Aneignungen und Simulacren von Simulacren. Gleichzeitig sei Rap als eine Form der Dekonstruktion zu verstehen:

»An instrumental track recorded in Memphis, combined with a back-up vocal from New York, and a lead voice from L.A. Rap simply continues this process of layered artistic composition by deconstructing and differently reassembling prepackaged musical products and then superimposing the MC's added layer of lyrics so as to produce a new work« (ebd.: 206).

Gegen Shustermans Thesen ist eingewandt worden, dass es den Musikern weniger um ein aus der europäischen Kultur stammendes avantgardistisches Kunstkonzept gehe, sondern um das Erschaffen von Musik, die »funktioniert«, d.h. für die Produzenten entsprechend ihrer Vorstellungen klingt, dadurch die »richtige « Atmosphäre entstehen lässt und die »groovt « (Schloss 2004: 63-78). Ein zweiter gegen Shustermans These vorgebrachter Einwand lautet, dass die Musik nicht postmodern, sondern afroamerikanisch sei. Dafür spricht, dass viele der im HipHop verwendeten Samples von afroamerikanischen Musikern stammen, dass Rap eine musikalische Form der Alltagssprache von Afroamerikanern ist und dass sich Techno in einem afroamerikanischen Umfeld entwickelt hat und über die Nähe zu House-Musik und Disco mit einer afroamerikanischen Szene in Verbindung steht (Hughes 1994). Aber in dieser Lesart steckt wiederum die Gefahr, Techno und Rap zu einer rein afroamerikanischen Kultur zu essentialisieren, durch die alle weiteren Einflussquellen und Weiterentwicklungen diskreditiert werden können.

Das Prinzip der Montage bietet aber noch eine weitere Lesart an, die die Musik wiederum eng mit dem Stadtraum in Verbindung bringt. Seit der Industrialisierung hat sich das Leben in den Städten beschleunigt und differenziert. Darüber hinaus sind die Großstädte durch globale Vernetzung und Migrationsbewegungen durch kulturelle Heterogenität geprägt (Hannerz 1980: 91-118). Unterschiedliche Lebensentwürfe, Einstellungen, Stile und Praktiken existieren in den Städten nebeneinander. Aber die Stadt ist auch der zentrale Ort, an dem diese unterschiedlichen Kulturen aufeinander treffen und sich nicht selten gegenseitig beeinflussen.

Diese beschleunigte Heterogenität, die das Stadtleben prägt, lässt sich am einfachsten durch Montagen darstellen. Sie ist das Prinzip, mit dem sich auf der formalen Ebene die rasanten Veränderungen der industrialisierten Stadträume in Kunst übersetzen lassen. Eine ganz ähnliche formale Übersetzungsarbeit lässt sich auch für das Montageprinzip in HipHop und Techno 
beobachten. Die Nutzung von Schallplatten und Plattenspieler als Musikinstrumente und die Verwendung von Samplern ermöglicht die schon von den Futuristen oder der musique concrète anvisierte Einbindung von Alltagsgeräuschen und dem gesamten Archiv gespeicherter Klänge in Musik. Mit der Montage wird es möglich, die in den Städten anzutreffenden Überlagerungen von Kulturen, Klängen und Praktiken in Musik darzustellen. Deshalb ist die Musik des HipHop oder Techno vor allem eines: städtisch.

\section{Rhythmus}

Lärm und Montage könnten zur These verleiten, dass Punkstücke, HipHopoder Techno-Tracks die klangliche Darstellung von Städten seien. Besonders für HipHop ist genau dies auch behauptet worden. Peter McLaren beschreibt zum Beispiel Rap als Möglichkeit eines fiktiven Besuchs von Innenstadtghettos:

»Through its cultural fusions, intercultural encounters, and expressive articulations, we are invited by gangsta rap to visit spaces we have never lived physically, nor would ever wish to - spaces that function significantly in the manufacturing of identity« (McLaren 1999: 47).

Ähnlich formuliert Tricia Rose die Qualität von HipHop:

»Hip Hop replicates and reimagines the experience of urban life and symbolically appropriates urban space through sampling, attitude, dance, style, and sound effects. Talk of subways, crews and posses, urban noise, economic stagnation, static and crossed signals leap out of hip hop lyrics, sounds, and themes« (Rose 1994: 22).

Aber auch wenn in Raptexten sehr oft das Leben in Städten beschrieben wird und sich direkte onomatopoetische Hinweise auf die Stadt in der Musik finden lassen, so stellt die Musik trotzdem keine tonmalerische Darstellung der Stadt dar. Dagegen spricht vor allem die immense Präsenz des Rhythmus, die sich nicht nur im HipHop, sondern auch im Punk und Techno beobachten lässt. Der Rhythmus ordnet und strukturiert die Musik, sodass sie gerade Eigenschaften besitzt, die dem städtischen Klangraum fehlen.

Der Rhythmus verweist aber negativ auf das Leben in Städten. Musik, die den Rhythmus in den Vordergrund stellt, bringt besonders leicht Menschen zum Tanzen (Frith 1996: 141-144; Middleton 1990: 139). Die Radikalisierung der Bedeutung des Rhythmus im Punk, HipHop und Techno macht diese Musiken deshalb zu idealen Medien der Vergemeinschaftung, also genau zu dem, was durch die Fragmentierung der Stadträume, der individuali- 
sierten Lebensweise ihrer Bewohner und den kontinuierlichen Umgang mit Fremden zusehends schwieriger wird.

\section{Fazit: Die Erzeugung einer idealisierten städtischen Atmosphäre}

Wenn die Musik kein Tonbild der Stadt abgibt, wie lässt sie sich dann charakterisieren und in welcher Verbindung steht sie dann zur Stadt? Wie sich zeigt, korrespondieren zumindest die ästhetischen Prinzipien des Lärms und der Montage direkt mit dem Stadtraum. Anders als Kivy dies für die europäische Kunstmusik postuliert, entsteht die strukturelle Isomorphie in der populären Musik nicht über Tonfolgen, sondern durch die Nähe der grundsätzlichen ästhetischen Prinzipien der Musik zu Stadträumen. Diese Isomorphie zwischen Musik und Stadt wird in Stilen wie Punk, HipHop oder Techno noch zusätzlich durch Texte über das Leben in Städten und Praktiken, die an den Stadtraum gebunden sind, unterstützt (Klein 2001, Friedrich 2005). Der Rhythmus lässt sich andererseits als Prinzip charakterisieren, das negativ mit dem kakophonen und Vergemeinschaftung erschwerenden Stadtraum korrespondiert. So kann man im Hören in eine perfekte und idealisierte Stadtatmosphäre eintauchen, die es an jedem Ort der Welt ermöglicht, ein intensives In-der-Stadt-Sein zu erleben. Darin ist auch ein Element für den Erfolg der drei Stile zu erkennen. Sie erlauben schon alleine im Hören etwas, was die fragmentierte und durch globale Flüsse geprägte urbane Welt immer schwieriger werden lässt: Verortung. Populäre Musik wie Punk, Techno oder HipHop lässt sich deshalb nicht nur als nicht-denotative Repräsentation der Stadt charakterisieren, sondern ist daran mitbeteiligt, eine urbane Kultur in der ganzen Welt zu konstituieren. Nicht nur Kleidungsstile und Praktiken, sondern die Musik selbst ist städtisch und prägt dadurch die Vorstellungswelten ihrer Hörer. 


\section{Literatur}

Adorno, Theodor W. (2003). Ästhetische Theorie. Hg. v. Rolf Tiedemann (= Gesammelte Schriften 7). Frankfurt/M.: Suhrkamp (2. Aufl.).

Behrens, Roger (1996). Pop Kultur Industrie. Zur Philosophie der populären Musik. Würzburg: Königshausen \& Neumann.

Blum, Elisabeth (2003). Schöne neue Stadt. Wie der Sicherheitswahn die urbane Welt diszipliniert. Basel, Boston, Berlin: Birkhäuser.

Burgin, Victor (1996). In/Different Spaces. Place and Memory in Visual Culture. Berkeley, Los Angeles, London: University of California Press.

DeNora, Tia (2003). After Adorno. Rethinking Music Sociology. Cambridge: Cambridge University Press.

Donald, James (1999). Imagining the Modern City. Minneapolis: University of Minnesota Press.

Fellerer, Karl Gustav (1989). »Der Futurismus in der italienischen Musik.« In: Ders., Studien zur Musik des 19. Jahrhundert. Band 4: Vom Musikdrama zum Futurismus. Regensburg: Bosse, S. 219-269.

Friedrich, Malte (2005). „।maginäres und Reales. Popmusik in der postindustriellen Stadt. «In: Stadt. Szenen. Künstlerische Praktiken und theoretische Positionen. Hg. v. Gabriele Klein. Wien: Passagen, S. 129-142.

Frith, Simon (1996). Performing Rites. On the Value of Popular Music. Oxford, New York: Oxford University Press.

George, Nelson (2002). $R$ \& B. Die Geschichte der schwarzen Musik. Freiburg: Orange Press.

Gillett, Charlie (1996). The Sound of the City. London: First Da Capo Press (3. Aufl.).

Gilloch, Grame (1996). Myth and Metropolis. Walter Benjamin and the City. Cambridge (UK), Oxford, Cambridge (USA): Blackwell.

Gracyk, Theodore (1996). Rhythm and Noise. An Aesthetics of Rock. Durham, London: I.B. Tauris.

Hannerz, Ulf (1980): Exploring the City. Inquiries Toward an Urban Anthropology. New York: Columbia University Press.

Hauser, Arnold (1967). Sozialgeschichte der Kunst und Literatur. München: Beck.

Hebdige, Dick (1979). Subculture. The Meaning of Style. London, New York: Routledge.

Hughes, Walter (1994). »In the Empire of the Beat. Discipline and Disco.« In: Microphone Fiends. Youth Music \& Youth Culture. Hg. v. Andrew Ross und Tricia Rose. New York, London: Routledge, S. 147-157.

Keyes, Cheryl L. (2002). Rap Music and Street Consciousness. Urbana, Chicago: University of Illinois Press.

Kivy, Peter (1980). The Corded Shell. Reflections on Musical Expression. Princeton: Princeton University Press.

Kivy, Peter (1991). Sound and Semblance. Reflections on Musical Representation. Ithaca, London: Cornell University Press.

Kivy, Peter (2001). Introduction to a Philosophy of Music. Oxford: Clarendon Press.

Klein, Gabriele (2001). »Urban Story Telling. Tanz- und Popkultur.« In: TechnoSoziologie. Erkundungen einer Jugendkultur. Hg. v. Ronald Hitzler und Michaela Pfadenhauer. Opladen: Leske + Budrich, S. 161-184. 
Krims, Adam (2003). »Marxist Music Analysis without Adorno. Popular Music and Urban Geography. «In: Analyzing Popular Music. Hg. v. Allan F. Moore. Cambridge: Cambridge University Press, S. 131-157.

Krims, Adam (2007). Music and Urban Geography. New York, London: Routledge.

Ludwig, Walter (2004). Musiksoziologie. Bern u.a.: Peter Lang.

Martin, Peter J. (1995). Sounds and Society. Themes in the Sociology of Music. Manchester, New York: Manchester University Press.

McLaren, Peter (1999). „Gangsta Pedagogy and Ghettocentricity. The Hip-Hop Nation as Counterpublic Sphere. In: Sound Identities. Popular Music and the Cultural Politics of Education. Hg. v. Cameron McCarthy, Glenn Hudak, Shawn Miklavicic und Paula Saukko. New York u.a.: Peter Lang, S. 19-95.

Middleton, Richard (1990). Studying Popular Music. Milton Keynes, Philadelphia: Open University Press.

Neumeyer, Harald (1999). Der Flaneur. Konzeptionen der Moderne. Würzburg: Königshausen \& Neumann.

Rose, Tricia (1994). Black Noise. Rap Music and Black Culture in Contemporary America. Hanover, London: Wesleyan University Press.

Rösing, Helmut (1999). "Soundscape - Urbanität und Musik. «In: 50 Jahre Musikwissenschaftliches Institut in Hamburg. Bestandsaufnahme - aktuelle Forschung - Ausblick. Hg. v. Peter Petersen und Helmut Rösing (= Hamburger Jahrbuch für Musikwissenschaft 16). Frankfurt/M. u.a.: Peter Lang, S. 113-125.

Schafer, R. Murray (1994). The Soundscape. Our Sonic Environment and the Tuning of the Word. Rochester (Vermont): Destiny Books (2. Aufl.).

Schloss, Joseph G. (2004). Making Beats. The Art of Sample-Based Hip-Hop. Middletown (Connecticut): Wesleyan University Press.

Shusterman, Richard (1992). "The Fine Art of Rap.« In: Pragmatist Aesthetics. Living Beauty, Rethinking Art. Oxford (UK), Cambridge (USA): Blackwell, S. 201235.

Georg Simmel (1995). Soziologie. Untersuchungen über die Formen der Vergesellschaftung (= Gesamtausgabe 11). Frankfurt/M.: Suhrkamp (2. Aufl.).

Vogt, Sabine (2005). Clubräume - Freiräume. Musikalische Lebensentwürfe in den Jugendkulturen Berlins. Kassel u.a.: Bärenreiter.

\begin{abstract}
Cities are not only material artefacts but also exist as representations. Through global media flows this representations influence cultures all over the world. Among the most influential sources of these representations are popular music scenes. In most cases studies that analyse these scenes authors concentrate on the practices, the visual styles and the values of the scenes, but have surprisingly little if anything to say about the music itself. In these contexts the representations of cities mean usually visual representations. This article argues that there is an audible connection between the city and popular music cultures, a connection which can be found e.g. in noise, montage and rhythm as urban aesthetic principles.
\end{abstract}

